\title{
Ubiquitinated deiodinase: not dead yet
}

\author{
Ronald J. Koenig \\ Endocrinology Division, Department of Internal Medicine, University of Michigan \\ Medical Center, Ann Arbor, Michigan, USA
}

Originating in the thyroid, the prohormone thyroxine is converted to triiodothyronine, which is essential in brain development, growth, and metabolism. A study in this issue (see the related article beginning on page 189) reveals a novel mechanism for controlling triiodothyronine production that provides the first example of enzyme activity being restored by deubiquitination.

J. Clin. Invest. 112:145-147 (2003). doi:10.1172/JCI200319157.

Thyroxine (T4), the major secretory product of the thyroid gland, is a prohormone. Removal of an outerring iodine by either type 1 or type 2 iodothyronine deiodinase (D1, D2) yields the biologically active hormone 3,5,3'-triiodothyronine (T3). The deiodinases are interesting enzymes. For example, their catalytic sites contain the rare amino acid selenocysteine, encoded by UGA, which under ordinary circumstances functions as a stop codon $(1,2)$.

D2 is particularly fascinating (see ref. 3 for an outstanding review). In rodents, it is expressed primarily in brown adipose tissue (BAT), brain, and pituitary, and also in the developing cochlea. In humans, D2 is expressed somewhat more broadly, including in skeletal muscle, heart, and thyroid. Approximately 50\% of nuclear T3 receptors (TRs) are ligand-occupied in most tissues of euthyroid rats. However, a substantially higher fraction of the TRs of

\footnotetext{
Address correspondence to: Ronald J. Koenig, University of Michigan Medical Center, 5560 Medical Science Research Building II, 1150 W. Medical Center Drive, Ann Arbor, Michigan 48109-0678, USA. Phone: (734) 763-3056; Fax: (734) 936-6684; E-mail: rkoenig@umich.edu.

Conflict of interest: The author has declared that no conflict of interest exists.

Nonstandard abbreviations used: thyroxine (T4); types 1 and 2 iodothyronine deiodinase (D1 and D2); 3,5,3'-triiodothyronine (T3); brown adipose tissue (BAT); T3 receptor (TR); ubiquitinated D2 (Ub-D2); ER-associated degradation (ERAD); von Hippel-Lindau protein-interacting deubiquitinating enzyme (VDU); von Hippel-Lindau protein (pVHL).
}

tissues that express D2 are ligandoccupied - from $75 \%$ in BAT to $97 \%$ in cerebral cortex. This extra T3 is generated locally by D2 from plasmaderived T4. In contrast, T3 generated by $\mathrm{D} 1$ does not have direct access to nuclei but instead must first be exported into the plasma. The difference appears to relate to the subcellular localization of these enzymes; D2 is an ER enzyme localized to the perinuclear space, whereas D1 lies in the plasma membrane (4). Thus, although both D1 and D2 contribute to the plasma T3, D2 provides an additional local source of nuclear $\mathrm{T} 3$. The consequence is that D2-containing organs can regulate their nuclear TR occupancy independent of the plasma T3 level. It follows, then, that local regulation of D2 activity is critically important in determining the thyroid status of D2-containing organs, and that plasma T4 is an important source of nuclear $\mathrm{T} 3$ in these organs.

\section{Acute regulation of $\mathrm{D} 2$ and adaptive thermogenesis}

BAT is an important site of heat generation in response to decreased ambient temperature (adaptive thermogenesis) in small mammals, including newborn humans. Cold exposure stimulates the sympathetic nervous system, which induces BAT uncoupling protein-1 (UCP-1), resulting in heat production. Hypothyroid rats are unable to mount an adequate BAT response to cold (5). This results partly from the fact that nor- epinephrine (via a cAMP response element) and T3 synergistically induce UCP-1 transcription. However, this synergism requires complete occupancy of BAT TRs. How does BAT get enough T3 to saturate its TRs? Norepinephrine rapidly induces BAT D2, generating $\mathrm{T} 3$ and causing local hyperthyroidism.

Unlike many hormones (insulin, for example), T3 circulates at essentially constant levels throughout the day. Thus, T3 has not been considered a hormone that responds acutely to metabolic perturbations. Yet, cold exposure is an example of such a perturbation, and the induction of BAT D2 provides the mechanism whereby an acute increase in local T3 can be achieved without substantially impacting circulating T3 levels.

The D2 enzyme has a short half-life of about 45 minutes, in contrast to the approximately 12-hour half-life of D1. Bianco and colleagues have shown previously that the short halflife of D2 is due to ubiquitination and proteasomal degradation, and that T4 catalysis further decreases the D2 half-life by enhancing the formation of ubiquitinated D2 (Ub-D2) (6). Many other ER proteins are subject to ubiquitination and proteasomal degradation, a process known as ERassociated degradation (ERAD), and it is common for ERAD to be regulated. For example, analogous to the enhancement of D2 ERAD by T4, HMG CoA reductase ERAD is stimulated by flux through the mevalonate pathway (7).

\section{Reactivation of Ub-D2 by deubiquitinases}

Although Ub-D2 is catalytically inactive, it exists in a netherworld, poised to be degraded in the black hole of the proteasome, or, potentially, to be reactivated by deubiquitination. In this issue of the JCI, Curcio-Morelli et al. (8) demonstrate that D2 interacts with von Hippel-Lindau protein-interacting deubiquitinating enzymes-1 and - 2 (VDU1 and VDU2), and that these interactions prolong the D2 half-life and rejuvenate enzyme activity by deubiquitinating 


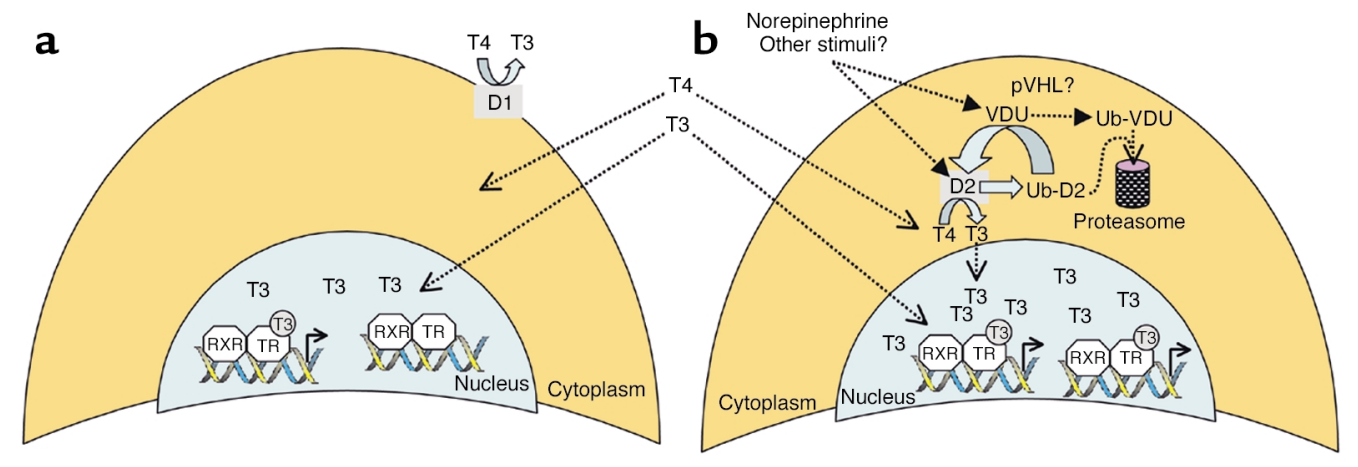

\begin{abstract}
Figure 1
Thyroid hormone metabolism and action in different cell types. (a) Hepatocytes (which express D1), as a model for cells that do not express D2. D1 converts plasma T4 to T3, but this T3 does not have direct access to the cell nucleus. Plasma T3 enters the nucleus. In the euthyroid state, TRs are about 50\% T3-occupied and target genes are induced proportionately. (b) BAT, as a model for tissues that express D2. Plasma T3 enters the nucleus. In addition, the ER enzyme D2 converts T4 to T3, which has direct access to the nucleus, resulting in a high level of TR occupancy by T3. Target genes are induced proportionately. D2 is regulated by metabolic perturbations, e.g., cold exposure (via norepinephrine) in BAT. The catalytic activity of D2 stimulates its ubiquitination and potential degradation in proteasomes. However, the VDUs can prevent degradation and recover enzyme activity by deubiquitinating Ub-D2. In BAT, VDU1 itself is induced by cold exposure, via norepinephrine. In some tissues, VDUs are ubiquitinated by PVHL and degraded, but this has not been studied in BAT. The retinoid $X$ receptor $(R X R)$ is the dimerization partner for $T R$.
\end{abstract}

Ub-D2. Remarkably, VDU1 is induced rapidly in BAT by cold exposure or norepinephrine, providing a new mechanism to achieve local saturation of TRs and hence an adequate thermogenic response to cold (Figure 1). Reactivation of Ub-D2 may be a particularly valuable mechanism for this selenoprotein, as it avoids the need to resynthesize D2. Incorporation of selenocysteine at UGA codons is inefficient and decreases translation efficiency about 100-fold (9).

D2 is the first specific substrate identified for the VDUs, and this is the first example of enzyme reactivation by deubiquitination. The VDUs are the first ubiquitin-specific processing enzymes shown to specifically deubiquitinate an ERAD substrate. What, actually, are the VDUs? The von Hippel-Lindau protein ( $\mathrm{pVHL}$ ) is a component of an E3 ubiquitin ligase that plays a role in determining substrate specificity for ubiquitination $(10,11)$. Mutations in the VHL gene are associated with the autosomal dominant tumor syndrome von Hippel-Lindau disease (characterized by CNS and retinal hemangioblastomas, renal clear cell carcinomas, pheochromocytomas, and others) as well as sporadic renal carcinomas. VDU1 was identified by yeast two- hybrid screen as a pVHL-interacting protein (12), and VDU2 was identified by homology to VDU1 (13). Both VDUs are ubiquitinated by the PVHL E3 ligase and are themselves deubiquitinases, suggesting that PVHL-mediated degradation of VDUs regulates the ubiquitin-proteasome pathway. Since PVHL stimulates degradation of VDUs, there is a potential link between pVHL (which is widely expressed) and decreased local T3 generation by $\mathrm{D} 2$.

\section{The broader picture}

Many important questions remain. For example, do other specific substrates exist for VDUs? Many structurally diverse deubiquitinases have been identified; do all have specific substrates? How does T4 stimulate D2 ubiquitination? The process is not specific to $\mathrm{T} 4$, as other $\mathrm{D} 2 \mathrm{sub}$ strates (reverse T3, for example) do the same, but it does require catalytically active enzyme.

Do VDUs reactivate Ub-D2 in tissues other than BAT, and if so, what regulates VDU activity in those tissues? VDU1 is expressed in other D2containing organs, including brain, heart, placenta, and skeletal muscle. One might consider situations, besides cold exposure, in which rapid changes in $\mathrm{T} 3$ production could be expected. For example, virtually any serious illness is associated with decreased plasma T3 and decreased T4-to-T3 conversion, the so-called sick-euthyroid syndrome (14). This syndrome may be an adaptive response to illness, as tissue hypothyroidism should decrease oxygen consumption and conserve limited cellular resources. Since D2 probably contributes a significant portion of plasma $\mathrm{T} 3$, a rapid decrease in $\mathrm{D} 2$ activity could contribute to the sickeuthyroid syndrome. One might speculate that cytokines or other general markers of illness could impair VDU activity and hence tip the balance toward proteasomal degradation of D2. Another situation to consider is brain development, which is critically dependent on local D2-generated T3 $(15,16)$. Perhaps D2 regulation by VDU might be expected here, with VDU activity being regulated by developmental factors. Importantly the local thyroid status of D2-containing tissues may not be reflected in the plasma thyroid-stimulating hormone $\mathrm{T} 4$, and $\mathrm{T} 3$ levels. There is no simple way to measure local thyroid status in humans, but it is easy to imagine that derangements in these processes could have important pathophysiological consequences. 
1. Berry, M.J., Banu, L., and Larsen, P.R. 1991. Type I iodothyronine deiodinase is a selenocysteinecontaining enzyme. Nature. 349:438-440.

2. Davey, J.C., Becker, K.B., Schneider, M.J., St. Germain, D.L., and Galton, V.A. 1995. Cloning of a cDNA for the type II iodothyronine deiodinase. J. Biol. Chem. 270:26786-26789.

3. Bianco, A.C., Salvatore, D., Gereben, B., Berry, M.J., and Larsen, P.R. 2002. Biochemistry, cellular and molecular biology, and physiological roles of the iodothyronine selenodeiodinases. Endocr. Rev. 23:38-89.

4. Baqui, M.M.A., Gereben, B., Harney, J.W., Larsen, P.R., and Bianco, A.C. 2000. Distinct subcellular localization of transiently expressed types 1 and 2 iodothyronine deiodinases as determined by immunofluorescence confocal microscopy. Endocrinology. 141:4309-4312.

5. Bianco, A.C., and Silva, J.E. 1987. Intracellular conversion of thyroxine to triiodothyronine is required for the optimal thermogenic function of brown adipose tissue. J. Clin. Invest. 79:295-300.

6. Gereben, B., Goncalves, C., Harney, J.W., Larsen, P.R., and Bianco, A.C. 2000. Selective proteolysis of human type 2 deiodinase: a novel ubiquitin proteasomal mediated mechanism for regulation of hormone activation. Mol. Endocrinol. 14:1697-1708.

7. Ravid, T., Doolman, R., Avner, R., Harats, D., and Roitelman, R. 2000. The ubiquitin-proteasome pathway mediates the regulated degradation of mammalian 3-hydroxy-3-methylglutaryl-coenzyme A reductase. J. Biol. Chem 275:35840-35847.

8. Curcio-Morelli, C., et al. 2003. Deubiquitination of type 2 iodothyronine deiodinase by von Hippel-Lindau protein-interacting deubiquitinating enzymes regulates thyroid hormone activation. J. Clin. Invest. 112:189-196. doi:10.1172/JCI200318348.

9. Berry, M.J., Maia, A.L., Kieffer, J.D., Harney, J.W., and Larsen, P.R. 1992. Substitution of cysteine for selenocysteine in type I iodothyronine deiodinase reduces the catalytic efficiency of the protein but enhances its translation. Endocrinology. 131:1848-1852

10. Latif, F., et al. 1993. Identification of the von Hippel-Lindau disease tumor suppressor gene. Science. 260:1317-1320.
11. Ohh, M., and Kaelin, W.G., Jr. 1999. The von Hip pel-Lindau tumour suppressor protein: new perspectives. Mol. Med. Today. 5:257-263.

12. Li, Z., et al. 2002. Ubiquitination of a novel deubiquitinating enzyme requires direct binding to von Hippel-Lindau tumor suppresor protein. J. Biol. Chem. 277:4656-4662.

13. Li, Z., et al. 2002. Identification of a deubiquitinating enzyme subfamily as substrates of the von Hippel-Lindau tumor suppressor. Biochem. Biophys. Res. Commun. 294:700-709.

14. De Groot, L.J. 1999. Dangerous dogmas in medicine: the nonthyroidal illness syndrome. J. Clin. Endocrinol. Metab. 84:151-164.

15. Crantz, F.R., Silva, J.E., and Larsen, P.R. 1982. An analysis of the sources and quantity of 3,5,3'-triiodothyronine specifically bound to nuclear receptors in rat cerebral cortex and cerebellum. Endocrinology. 110:367-375.

16. Campos-Barros, A., et al. 1996. Phenolic and tyrosyl ring iodothyronine deiodination and thyroid hormone concentrations in the human central nervous system. J. Clin. Endocrinol. Metab. 81:2179-2185

\title{
Osteopontin: a bridge between bone and the immune system
}

\author{
Ellen M. Gravallese
}

Beth Israel Deaconess Medical Center, Harvard Institutes of Medicine, Boston, Massachusetts, USA

\begin{abstract}
The molecular mechanisms underlying the putative role of osteopontin in the chronic inflammatory disease rheumatoid arthritis are unclear. A study in a murine model of arthritis now demonstrates that a specific antibody directed against the exposed osteopontin epitope SLAYGLR (see the related article beginning on page 181) is capable of preventing inflammatory cell infiltration in arthritic joints.
\end{abstract}

\section{J. Clin. Invest. 112:147-149 (2003). doi:10.1172/JCI200319190.}

In recent years a number of studies have linked factors involved in inflammation to those critical for bone physiology and remodeling. One well described story is that of the receptor-activator of NF- $\kappa \mathrm{B}$ ligand (RANKL)/osteoprotegerin (OPG) system, which plays a role in the

\footnotetext{
Address correspondence to: Ellen M. Gravallese, Beth Israel Deaconess Medical Center, Harvard Institutes of Medicine, Room 241, 4 Blackfan Circle, Boston, Massachusetts 02115, USA.

Phone: (617) 667-0717; Fax: (617) 975-5299; E-mail: egravall@bidmc.harvard.edu.

Conflict of interest: The author has declared that no conflict of interest exists.

Nonstandard abbreviations used: receptoractivator of NF- $\mathrm{KB}$ ligand (RANKL); osteoprotegerin (OPG); osteopontin (OPN); early $\mathrm{T}$ cell activation gene- 1 (Eta-1); rheumatoid arthritis (RA); collagen-induced arthritis (CIA); collagen antibody-induced arthritis (CAIA).
}

interaction between dendritic cells and $\mathrm{T}$ cells in the immune system and is the critical regulatory system for bone remodeling under physiologic conditions $(1,2)$. The RANKL/ OPG system is also likely to play an important role in several forms of pathologic bone loss, including that seen in osteoporosis, certain forms of cancer, and inflammatory arthritis (3). There is mounting evidence for the role of another cytokine, osteopontin (OPN; "bone-bridging" protein), also known as early $\mathrm{T}$ cell activation gene-1 (Eta-1), in providing a link between the immune system and bone. In this issue of the JCI, Yamamoto et al. (4) provide important new evidence indicating a role for OPN in the pathogenesis of inflammatory arthritis and associated joint destruction.

\section{The role of OPN in bone} and in the immune system

OPN is a phosphorylated glycoprotein secreted by activated macrophages, leukocytes, and activated $\mathrm{T}$ lymphocytes, and present in extracellular fluids, at sites of inflammation, and in the ECM of mineralized tissues $(5,6)$. This cytokine mediates important cellmatrix and cell-cell interactions. OPN is abundant in bone, where it facilitates the attachment of osteoclasts to the bone matrix via an interaction with cell surface $\alpha v \beta 3$ integrin and CD44, the hyaluronic acid receptor (7). $\mathrm{OPN}^{-/}$ mice have a subtle bone phenotype, with delayed and impaired bone resorption (7). In the immune system, OPN plays a role in chemotaxis, leading to the migration of macrophages and dendritic cells to sites of inflammation. Activation of $\mathrm{T}$ lymphocytes results in an increase in OPN transcription, hence its alternative designation as Eta- 1 . Weber et al. have demonstrated that OPN is a $\mathrm{T}$ lymphocyte suppressor factor and that it enhances B lymphocyte $\mathrm{Ig}$ production and proliferation (8). In addition, OPN is an important cytokine mediating Th1 immunity (9).

OPN interacts with a variety of cell surface receptors, including the $\alpha v \beta 3$, $\alpha v \beta 5, \alpha v \beta 1, \alpha 4 \beta 1, \alpha 8 \beta 1$, and $\alpha 9 \beta 1$ integrins, as well as CD44. Binding of OPN to these cell surface receptors stimulates cell adhesion, migration, and specific signaling functions. The major integrinbinding site in OPN is the arginineglycine-aspartate (RGD) integrin-binding motif, which is required for the 